\title{
Magnetohydrodynamic Fast Shocks and Their Relation to Solar Energetic Particle Event Intensities
}

\author{
Kan Liou ${ }^{1}$, Chin-Chun $\mathrm{Wu}^{2}$, Murray Dryer ${ }^{3}$, Shi-Tsan $\mathrm{Wu}^{4}$, Daniel B. Berdichevsky ${ }^{5}$, Simon Plunkett ${ }^{2}$, \\ Richard A. Mewaldt ${ }^{6}$, and Glenn M. Mason ${ }^{1}$ \\ ${ }^{1}$ The Johns Hopkins University Applied Physics Laboratory, Laurel, Maryland, USA \\ ${ }^{2}$ Naval Research Laboratory, Washington, DC, USA \\ ${ }^{3}$ NOAA Space Weather Prediction Center, Boulder, Colorado, USA (retired) \\ ${ }^{4}$ Center for Space Plasma and Aeronomic Research, University of Alabama, Huntsville, Alabama, USA \\ ${ }^{5}$ University of the District of Columbia, Washington, DC, USA \\ ${ }^{6}$ Space Radition Laboratory, Caltech, Pasadena, California, USA
}

Received 19 December 2011, accepted 8 May 2012

\begin{abstract}
Gradual solar energetic particles (SEPs) are associated with interplanetary (IP) shock driven by coronal mass ejections. Testing theories/models that are built around shock acceleration mechanisms is difficult due to the complexity of SEP fluxes acquired by single-point measurements. To circumvent this, we correlate fast-forward shock Mach numbers derived from a 1.5D magnetohydrodynamics simulation with the intensity of solar energetic oxygen $(\mathrm{O})$ and helium-4 $\left({ }^{4} \mathrm{He}\right)$ particles acquired by instruments aboard the ACE spacecraft during a series of coronal mass ejections in 2003 (October 28 - 31). A good correlation at the $5 \%$ significance level is found for $\mathrm{O}$ and ${ }^{4} \mathrm{He}$ with energy $(E)>\sim 10 \mathrm{MeV} \mathrm{n}^{-1}$, with the peak correlation coefficient $r=0.82$ for $\mathrm{O}\left(E=63.8-89.8 \mathrm{MeV} \mathrm{n}^{-1}\right)$ and $r=0.77$ for ${ }^{4} \mathrm{He}\left(E=18.0-29.4 \mathrm{MeV} \mathrm{n}^{-1}\right)$, respectively, for hourly averaged data. This result not only bolsters the causal relationship between IP fast shocks and SEPs, but also suggests that the Mach number of IP shocks is one of the major controlling parameters for the intensity of SEPs measured in the near-Earth space.
\end{abstract}

Key words: Solar energetic particles, CME driven-shocks, MHD simulation, ACE

Citation: Liou, K., C. C. Wu, M. Dryer, S. T. Wu, D. B. Berdichevsky, S. Plunkett, R. A. Mewaldt, and G. M. Mason, 2013: Magnetohydrodynamic fast shocks and their relation to solar energetic particle event intensities. Terr. Atmos. Ocean. Sci., 24, 165-173, doi: 10.3319/TAO.2012.05.08.01(SEC)

\section{INTRODUCTION}

Solar energetic particle (SEP) events are enhancements of high energy electrons, protons, and heavy ions, with energies ranging from tens of $\mathrm{keV}$ to $\mathrm{GeV}$, streaming outward from its source associated with solar flares and coronal mass ejections (CMEs). Studies of SEPs in the interplanetary space provide remote diagnosis of the source mechanisms and transport of these particles. Solar energetic particles (SEPs) also play a major role in space weather forecasting since it takes about only an hour for $10 \mathrm{MeV}$ protons to propagate from the Sun to the Earth and much more than that in the case of energetic storm particles (ESPs) since intensities often peak at the shock passage at 1 astronomical

\footnotetext{
* Corresponding author

E-mail:kan.liou@jhuapl.edu
}

unit (AU). Understanding the causes and to be able to predict the arrival of SEPs and ESPs are of importance in the space era because these high energy episodes pose threats to space vehicles and the health of astronauts. However, interpreting the intensity-time profile of in situ SEP data has ambiguities because of the following three fundamental problems. First, single point measurements at spacecraft cannot distinguish between spatial and temporal variations. Second, for a single observer, temporal and spatial variations of the source of energetic particles are not measurable. Finally, the structure of the shock and interplanetary magnetic field (IMF) connecting shock to the observers are not measureable. Nonetheless, studies of SEPs from data collected in space during the last few decades have generally established a qualitative picture of the SEP time-intensity profile morphology (see the review by Reames 1999). A concept of 
"two-class" ("impulsive" and "gradual") of SEP events is now largely accepted. Impulsive SEPs are associated with solar flares (e.g., Pallavicini et al. 1977), whereas the occurrence of gradual SEPs is well correlated with CMEs (e.g., Kahler et al. 1978).

Mechanisms responsible for accelerating particles to high energies are not well understood and studies of the acceleration mechanisms continue. Previous studies show that there is a close association between SEP events and slowdrifting Type II radio bursts (Wild et al. 1963). There is a good correlation between proton $\left(>2 \mathrm{MeV} \mathrm{n}^{-1}\right)$ peak intensities and the speed of their associated CME (Kahler 2001); the SEP peak flux (>10 MeV) is better correlated with the CME speed than with the X-ray flare peak flux (Gopalswamy et al. 2003). These results, along with many other studies suggest that large gradual SEP events are accelerated at the interplanetary shocks driven by CMEs. However, as shown in Kahler (2001), there is a large spread ( 3 decades) in the SEP proton flux for a given CME speed. Other parameters might be of more importance in controlling SEP fluxes at $1 \mathrm{AU}$. The use of CME speed as a proxy for the CME-driven shock is obviously inadequate since it is the CME speed in the solar wind reference frame that determines if a shock wave can be excited by the CME. Notice that the fast-mode shock Mach number is defined as the ratio of the upstream plasma flow speed in the shock frame to the upstream fastmode wave speed. The square of the shock Mach number is, to a first order approximation, equivalent to the ratio of the kinetic energy of the plasma in the shock frame to the sum of the thermal energy and the magnetic energy upstream of the shock, and can be considered as a measure of the free energy of the magnetized upstream plasma that can be converted by the shock to other forms of energy. Therefore, one can expect that a larger Mach number (more free energy) will result in higher SEP fluxes. Here we propose that the fast shock Mach number is a better proxy of the shock strength and we will demonstrate our view by correlating in situ SEP data with fast shock Mach number. Because of limited spacecraft coverage, shock surface regions of a given Mach number intensity are only observationally available in a fragmentary form (see, e.g., Berdichevsky et al. 2009). We will use shock Mach numbers derived from a magnetohydrodynamic (MHD) simulation to perform the analysis.

\section{DATA ANALYSIS}

\subsection{Observations of SEPs}

This study focuses on the time period from day 298 (October 25) to day 304 (October 31) of 2003, a time segment of the well-known active Halloween 2003 epoch (Dryer et al. 2004). During this period, enhancements of $\mathrm{MeV} \mathrm{n}^{-1}$ Helium-4 $\left({ }^{4} \mathrm{He}\right)$ and oxygen $(\mathrm{O})$ ions were observed by the Solar Isotope Spectrometer (SIS) (Stone et al. 1998) and the Ultra-Low-Energy Isotope Spectrometer (ULEIS) (Mason et al. 1998) on board the Advanced Composition Explorer (ACE) spacecraft on day 299 (October 26), day 301 (October 28), and day 303 (October 30). Figure 1a shows hourly averages of the differential energy flux for ${ }^{4} \mathrm{He}$ in 8 energy channels that cover $3.43-41.2 \mathrm{MeV} \mathrm{n}^{-1}$ energy range (see the top part of Fig. 1a's upper panel) and for $\mathrm{O}$ in 11 energy channels in which $3\left(1.28-10.4 \mathrm{MeV} \mathrm{n}^{-1}\right)$ are from ULEIS and $8\left(7.30-89.8 \mathrm{MeV} \mathrm{n}^{-1}\right)$ from SIS. All three events reveal a usual velocity dispersion effect. The rise time for the first two ${ }^{4} \mathrm{He}$ and $\mathrm{O}$ enhancements were rather short $(\sim 3 \mathrm{hr})$ relative to that for the third event $(\sim 10 \mathrm{hr})$. The sudden rise of the $\mathrm{MeV}{ }^{4} \mathrm{He}$ and $\mathrm{O}$ fluxes approximately coincides with bursts of large solar X-ray flares that occurred at 1735 UT on day 299 (Class: X1.2/3N at N05W33), 1102 UT on day 301 (Class: X17.2/4B at S16E08), and 2042 UT on day 302 (Class: X10/2B at S15W02). A series of flare onsets during this time, day 298 - 304, are marked in Fig. 1b. All three flares were followed by CMEs. ACE was magnetically connected to the observed initial flare sites in the first SEP event but not in the second and third SEP events (Mewaldt et al. 2005).

Three fast-mode IP shocks were observed by ACE at 0150 UT on day 301,0600 UT on day 302, and 1620 UT on day 303 (marked as vertical dashed lines in Fig. 1a). The arrival of the second and third IP shocks at ACE coincides with the peak low-energy SEP fluxes seen at the second and third events. A dramatic decrease in the SEP flux, especially the higher energy ${ }^{4} \mathrm{He}$ and $\mathrm{O}$, is found to follow immediately after the passage of the second IP shock.

\subsection{Shock Simulations}

Evolution of the flare-associated CME-driven shocks from the surface of the Sun out to 1 AU for the Halloween storm event has been studied and reported by $\mathrm{Wu}$ et al. $(2005,2006,2007)$. The simulation used a 1.5D adapted code (Panitchob 1987) to solve numerically a set of governing equations that satisfy the conservation of mass, momentum, and energy for a compressible, non-viscous, perfectly conducting, adiabatic $(\gamma=5 / 3)$ magneto-hydrodynamic fluid in the presence of gravitational forces between the Sun and the solar wind plasma. The simulation inputs (plasma density, temperature, and velocity associated with pressure pulses) were tuned to match the timing and the velocity profiles of the 4 IP fast shocks arriving at the ACE orbit. The shock Mach numbers are obtained directly from their reported result and are used to correlate with the observed SEP fluxes. Our use of the $1.5 \mathrm{D}$ approximation for the present study assumes that the shock origin is near the central meridian and that the magnetic connection to ACE is good; given the flare locations at W33, E08 and W02, the assumption is reasonable.

Figures $1 \mathrm{~b}$ and $\mathrm{c}$ show the simulation results from $\mathrm{Wu}$ et al. (2006). The fast-mode shock Mach numbers shown 
in Fig. $1 \mathrm{~b}$ are defined as $M_{f}=\left(V_{S}-V_{S W}\right) / C_{f}$, where $V_{S}$ is the shock speed in a fixed frame, $V_{S W}$ is the background solar wind speed, $C_{f}=(1 / 2)\left\{C_{s}^{2}+V_{A}^{2}+\left[\left(C_{s}^{2}+V_{A}^{2}\right)^{2}-4 C_{s}^{2} V_{A}^{2}\right.\right.$ $\left.\left.\cos ^{2} \theta_{B n}\right]^{1 / 2}\right\}$ is the magnetosonic fast-mode wave speed, $C_{s}=(\gamma p / \rho)^{1 / 2}$ is the sound speed, $V_{A}=|\boldsymbol{B}| /(4 \pi \rho)^{1 / 2}$ is the
Alfvén speed, and $\theta_{B n}$ is the angle between the upstream magnetic field $(\boldsymbol{B})$ and the shock normal. The shock wave speed was calculated as the time rate of the change of the shock location and the shock speed Mach number was computed by the wave transit method (Wu et al. 1996). In Wu

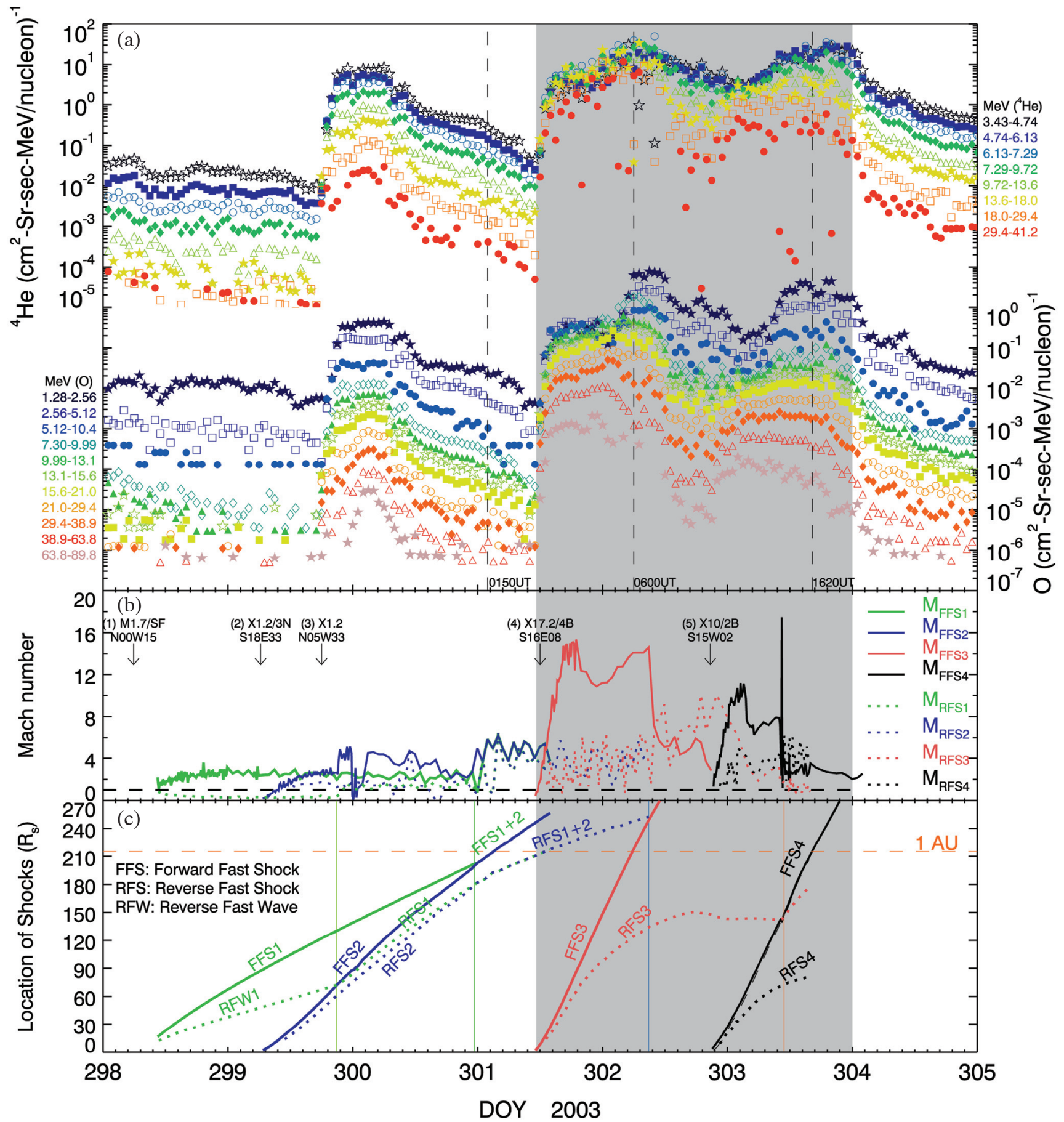

Fig. 1. (a) Time history of solar energetic particle differential energy fluxes observed by instruments on board the ACE spacecraft for ${ }^{4} \mathrm{He}(8$ energy channels: $3.43<E<41.2 \mathrm{MeV} \mathrm{n}^{-1}$, see insert for details) and $\mathrm{O}\left(8\right.$ channels: $7.30<E<89.8 \mathrm{MeV} \mathrm{n}^{-1}$ ) during the period from 25 October (day 298 ) to 1 November (day 305) in 2003. Three dashed lines mark the arrival time of the interplanetary shocks at ACE. (b) Mach numbers for fast-mode shock waves, 4 forward (FFS) and 4 reverse (RFS), based on 1.5D MHD simulation results (Wu et al. 2006). The insert shows 5 X-ray flare onsets (indicated by down arrows). (c) Distance of the simulated fast-mode shocks from the Sun. Four vertical lines show the times of shock-shock interactions. 
et al. (2006), three interplanetary shocks and one velocity jump observed by ACE were simulated using the 1.5 MHD code (Panitchob 1987), with their corresponding CME drivers launched at the Sun at the times of the four solar flares $(1,2,4$, and 5 marked in Fig. 1b). For each CME, a pair of fast-mode shocks are driven: one forward and one reverse. As indicated in Fig. 1c, a faster forward fast-mode shock (FFS) can catch up and subsequently merge with a slower FFS or a reverse fast-mode shock (RFS) to become a stronger one (e.g., FFS1 + FFS2 and RFS1 + RFS2). On the other hand, two shocks (one forward and one reverse) can collide and penetrate each other. The strength of the shock can become increasing (FFS2) or decreasing (FFS4) depending on their upstream and downstream solar wind conditions $(\mathrm{Wu}$ et al. 2006).

It is shown that the strength of shocks as indicated by their Mach numbers can vary significantly after they are created and propagated outward from the Sun. If SEP particles are accelerated by IP shocks, changes of the shock strength should appear in SEP data, i.e., a good correlation between the SEP flux and shock Mach numbers is expected. A quick comparison of the forward shock Mach number (FFS3 and FFS4) with the SEP fluxes seems to support such an idea. It is worth mentioning that the sharp decrease in SEP fluxes after the second shock arrival (on day 302) is probably caused by the sudden decrease in the shock Mach number, which drops substantially from 15 to 8 in a matter of 1 hour, resulting from the collision of the reverse fast-mode shock $(\mathrm{RFS} 1+2)$ with the forward fast-mode shock (FFS3).

To quantify the SEP flux-shock Mach number relationship, we correlate the simulated shock Mach numbers with the hourly averages of SEP data for the second and third SEP events. Note that we will omit consideration of the first SEP event since the first shock event, which is probably responsible for the first SEP event, was not studied by Wu et al. (2006). The other SEP events, in our opinion, will provide sufficient evidence for this study. We first resample the Mach number data to the hourly SEP data time grids. Then we calculate the Pearson product-moment correlation coefficient between the hourly Mach numbers and the logarithmic (10-based) SEP fluxes for the time period between day 301.47 and day 304 (shaded region shown in Fig. 1). Figure 2a shows the best correlation for ${ }^{4} \mathrm{He}(r=0.67)$ that happens to the $17.96-29.35 \mathrm{MeV} \mathrm{n}^{-1}$ energy channel following the fourth flare (see Fig. 1b) and the FFS/RFS interaction. The correlation coefficient can be much larger $(r=0.78)$ if the two outliers at the bottom of the Fig. 2a are removed. Figure $2 b$ shows correlation coefficients for all $8{ }^{4} \mathrm{He}$ energy channels. In general, the correlation is better for larger energy ${ }^{4} \mathrm{He}$ because it takes finite times for lower energy ${ }^{4} \mathrm{He}$ to propagate from the shock site to ACE. Thus, the correlation coefficients are somewhat lower for the other, lower energy ${ }^{4} \mathrm{He}$. To take this propagation effect into account, we estimate, to a first order approximation, the propagation delays of the ${ }^{4} \mathrm{He}$ particles using a "ballistic model." We divide the distance between the shock location and ACE by the speed of ${ }^{4} \mathrm{He}$ for the given energies for each time grid. Note that this simple time correction does not consider particle motion in a realistic magnetic field, particle scattering, and the finite time of particle acceleration, thus it underestimates the delay time. We believe that an underestimation should be negligible for the hourly data set used in this analysis. The delay time is added to the time grid and the flux at this new time is linearly interpolated and used to compute the correlation coefficients. The result is shown in Fig. 2b as filled squares. One can see improvements in the correlations except for the lowest energy channel. The peak correlation is $r=0.77$ for the 17.96 $20.35 \mathrm{MeV} \mathrm{n}^{-1}$ channel.

To test the significance of the correlation, the commonly used Student's-t test may not be appropriate because it assumes the randomness of data. Here we will first check the randomness of the data. Taking the data shown in Fig. 2a for ${ }^{4} \mathrm{He}\left(E=17.96-29.35 \mathrm{MeV} \mathrm{n}^{-1}\right)$ as an example, we plot the correlogram, which is a plot of sample autocorrelations versus time lags in Fig. 3. At zero lag, the correlation coefficient is 1 ; at nonzero lag time, the correlation coefficients decrease. After lag 6, the correlation coefficient falls below the $95 \%$ confidence level (defined by regions between two horizontal dashed lines) for a random series. Therefore, the number of independent points is $\sim 57 / 6 \sim 9$, which is much less than the original samples. Inspection of other data of different energy ranges indicates that the autocorrelation time is mainly in the $6-8 \mathrm{hr}$ range. Therefore, special care must be taken in dealing with the statistical significance of the correlation.

Here we will use the Random Phase technique developed by Ebisuzaki (1997) to test the statistical significance of correlation between two time series. The Random Phase technique is a non-parametric method and is similar to the Monte Carlo significance test in that both methods calculate cross correlation between a randomly reordered one of the time series with a second original series for a large number of times. However, there is a difference in generating the random time series. The Monte Carlo significance test method preserves the distribution of the original data, whereas the Random Phase method preserves the power spectra of the time series. The null hypothesis that the two series are uncorrelated can be rejected with the 0.05 significance level if the cross correlation computed is outside the $(0.025,0.975)$ quantiles of the empirical distribution of the cross-correlations.

The red dotted lines shown in Fig. $2 b$ are critical cross correlation coefficients at the 0.05 significance level derived from the Random Phase method using 10000 simulations, meaning that one can reject the null hypothesis at the 0.05 probability level whenever the cross correlation is greater than the critical value. The computed Pearson correlation 
coefficients are significant at the 0.05 probability level for ${ }^{4} \mathrm{He}$ having $E>20 \mathrm{MeV} \mathrm{n}^{-1}$ (the last two energy channels) and for propagated ${ }^{4} \mathrm{He}$ having $E>10 \mathrm{MeV} \mathrm{n}^{-1}$ (the last 4 highest energy channels).

We perform the same statistical analysis for $\mathrm{O}$ and the result is shown in Figs. 2c and d. The overall correlations for $\mathrm{O}$ are better than those for ${ }^{4} \mathrm{He}$. The largest correlation coefficient is $r=0.82$ (see Fig. 2c). After correcting for the propagation delay, the correlation is slightly improved. The poorer correlation of the Mach numbers and SEP intensities at lower energies is most likely due to the fact that the low energy particles often peak when the shock passes $1 \mathrm{AU}$ due to trapping of the particles near the shock (e.g., van Nes et al. 1984 and Desai et al. 2004). As Fig. 2d indicates, the correlations between high energy $\mathrm{O}\left(>\sim 10 \mathrm{MeV} \mathrm{n}^{-1}\right)$ fluxes and shock Mach number are statistically significant at the $5 \%$ probability level or better, irrespective to propagated or non-propagated oxygen SEP fluxes.

The good correlation between SEP fluxes and the fastmode shock Mach number illustrated in Fig. 2 prompts a question of whether the shock speed or the variation in wave speed is more important. Here we also perform similar correlation analysis and the result is shown in Fig. 4. The green dash-dotted line represents the 95\% confidence level derived from the Random Phase technique (Ebisuzaki 1997) and shows that SEP fluxes are better correlated with the
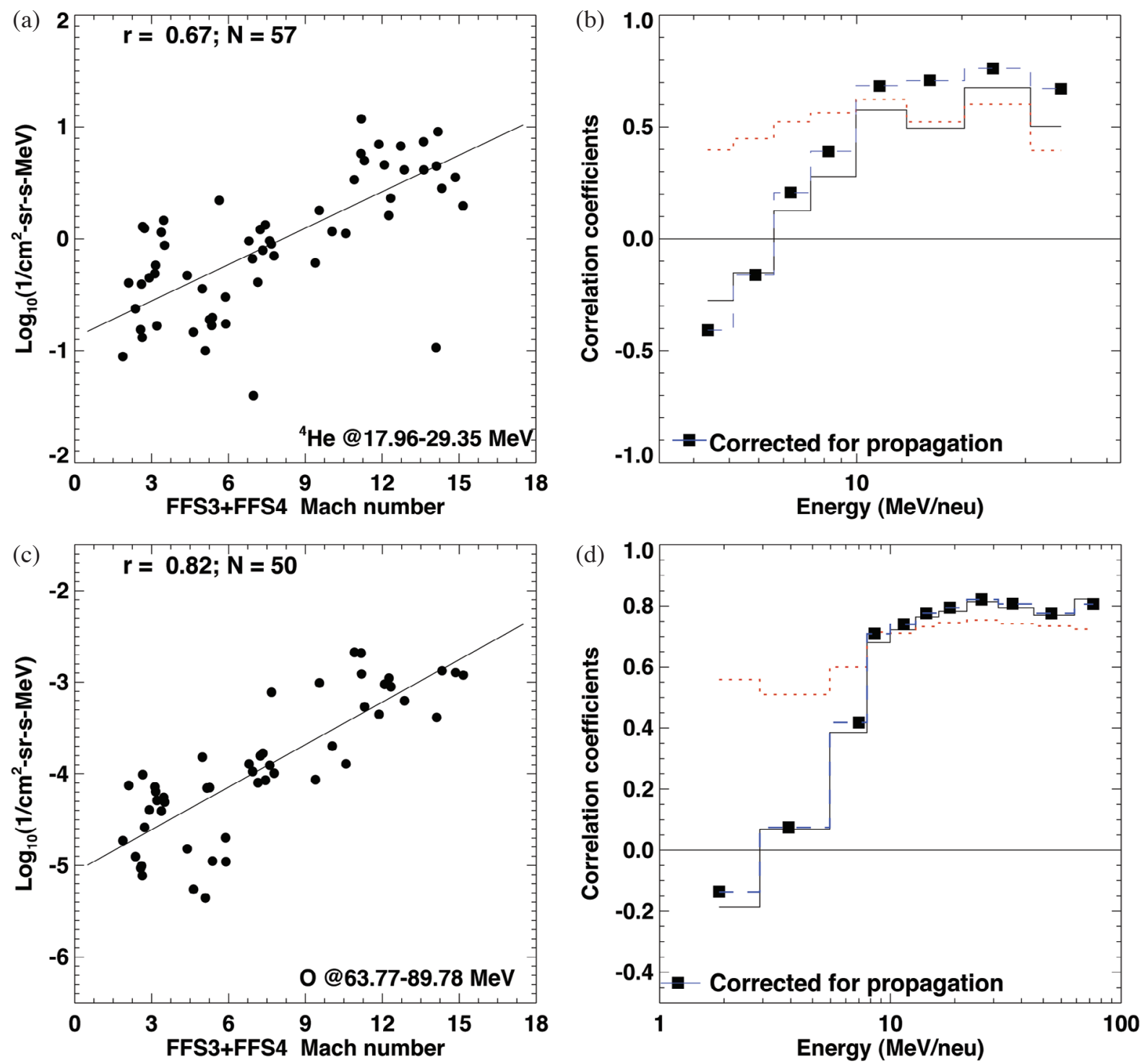

Fig. 2. (a) A scatter plot showing correlation between log differential flux of ${ }^{4} \mathrm{He}$ at $17.96-29.35 \mathrm{MeV} \mathrm{n}^{-1}$ channel and the Mach number of fastmode forward shocks (FFS3 and FFS4) for the time period from day 301.47 to day 304 of 2003, corresponding to the shaded area in Fig. 1. (b) Correlation coefficients between fast-mode shock Mach numbers and non-corrected/corrected (black solid lines/blue dashed lines + filled squares) logarithmic ${ }^{4} \mathrm{He}$ differential flux for all energy channels. Plotted in (c) and (d) are for $\mathrm{O}$ with the same format as (a) and (b), respectively, for ${ }^{4} \mathrm{He}$ Red dotted lines in (b) and (d) represent critical correlation coefficients at the 5\% significance level for given data points for sampled energy bins. 
Mach number (black) than with either the shock speed (red dotted line) or fast-mode wave speed (blue dashed line).

\section{DISCUSSION}

A good correlation between the CME-driven shocks and the intensity of SEP fluxes may not be unexpected as shock acceleration has long been considered as the primary mechanism for large, gradual, and long lasting SEP events. The present study of two SEP events indicates that up to

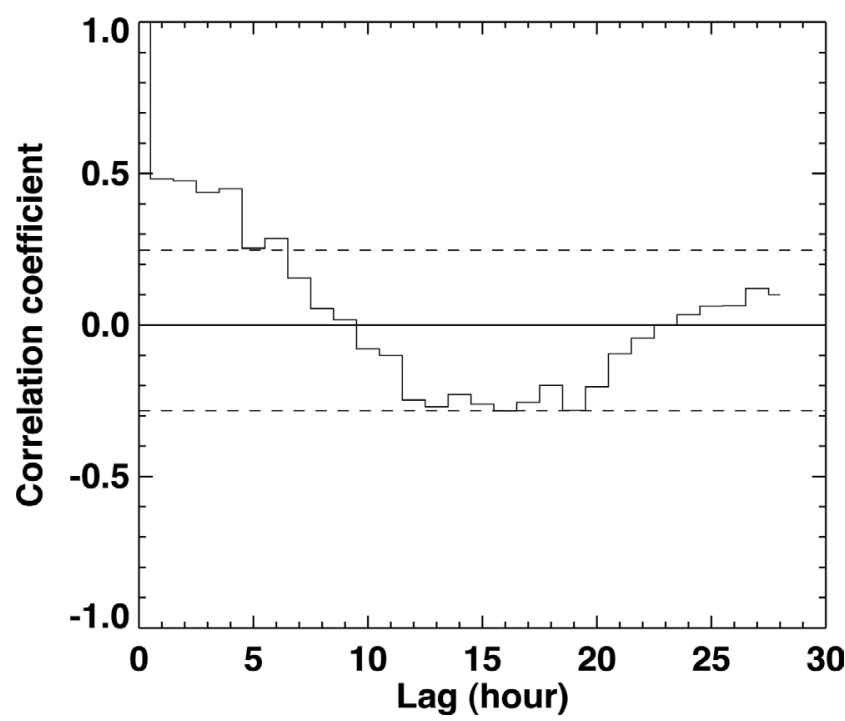

Fig. 3. Correlogram showing the autocorrelation coefficients as a function of time lags. The $95 \%$ confidence limits (approximated by $-1 / \mathrm{N}$ $\pm 2 / N^{1 / 2}$, where $N$ is the number of samples) are plotted as two dashed lines. $\sim 40 \%$ ( $60 \%$ after correcting for a scatter-free propagation) of the variability in ${ }^{4} \mathrm{He}$ flux and up to $\sim 70 \%$ of the variability in $\mathrm{O}$ flux are linked to the variability in the fast shock Mach number. This suggests that the fast shock Mach number is one of the major controlling parameters for the intensity of SEPs. If one assumes that particles are accelerated by shocks, the intensity of SEP flux must, to some extent, depend on the intensity of shock such as the Mach number. The square of the Mach number is proportional to $\sim V^{2} /\left(C_{s}^{2}+V_{A}^{2}\right)=\frac{1}{2} \rho V^{2} /\left(\frac{1}{2} \gamma P+B^{2} / 8 \pi\right) \sim \varepsilon_{K} /\left(\varepsilon_{T H}+\varepsilon_{B}\right)$, where $\varepsilon_{K}, \varepsilon_{T H}$, and $\varepsilon_{B}$ are the kinetic, thermal, and magnetic energy density of the plasma flow upstream of a perpendicular shock, and is proportional to $\sim \varepsilon_{K} / \varepsilon_{T H}$ or $\varepsilon_{K} / \varepsilon_{B}$ whichever is smaller for a parallel shock (the proportional form comes in between for oblique shocks). While it is believed that much of the kinetic energy of the upstream plasma flow will be converted to the thermal energy of plasma and magnetic turbulence downstream of the shock, a portion of the energy will go to energetic particles. No matter how the energy is converted, it is reasonable to assume that shocks with a larger Mach-number will result in SEP events with higher fluxes, as it is suggested by the present study result.

Another way to view the results herein is that the leading theory for shock acceleration relates the shock compression ratio with the SEP spectrum. The shock compression ratio is a function of the shock Mach number and the plasma beta of the upstream plasma (Ellison and Ramaty 1985). Therefore, a large Mach number would predict a harder SEP spectrum and a larger high-energy particle flux.

Previous observations have shown a trend that the peak fluxes of SEP events increase with CME speed; however a large scatter (a few decades) in the SEP peak fluxes is
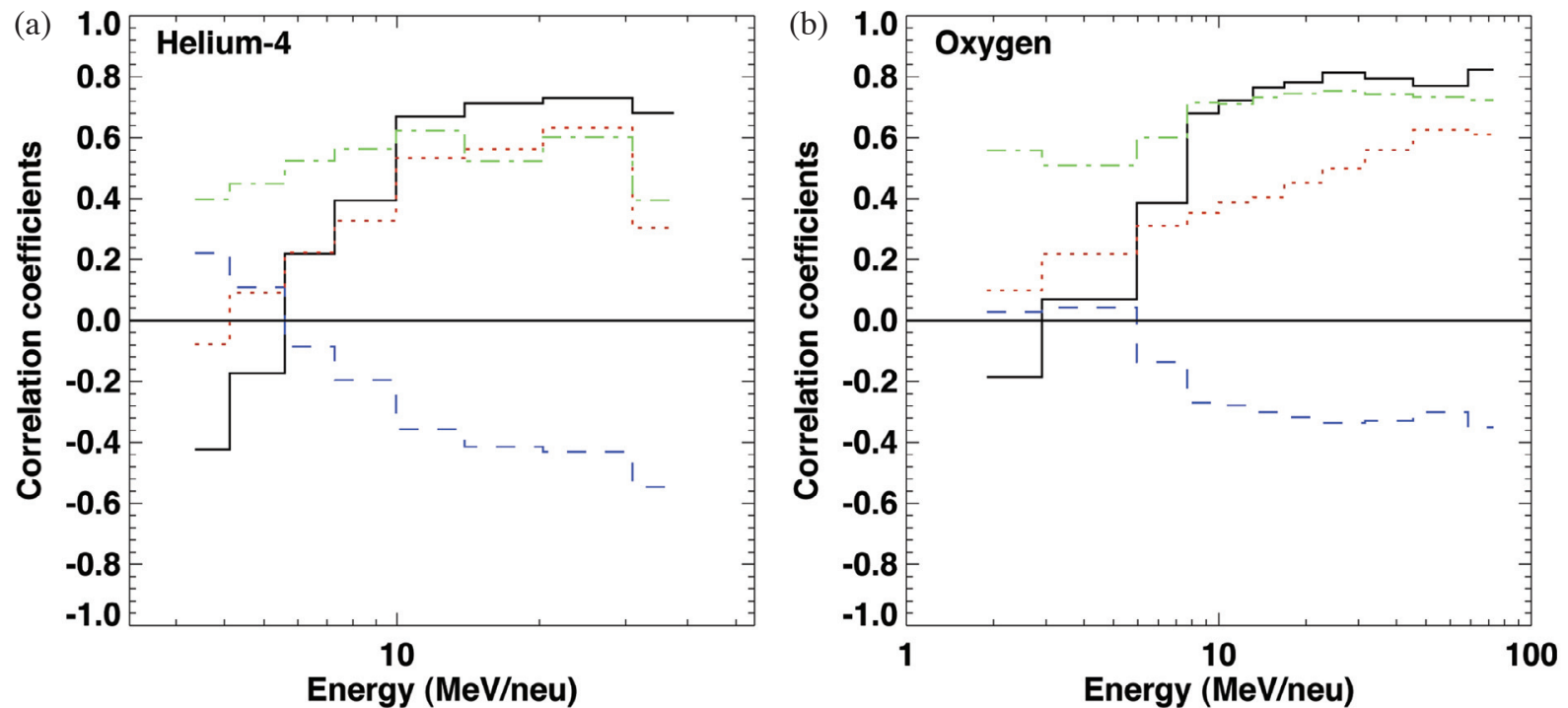

Fig. 4. Similar to the format used in Figs. 2 b and d. Here in both panels, (a) for He4 and (b) for O, black lines are for the fast-mode shock, red dotted lines are for the shock speed in the solar wind frame, blue dashed lines are for the fast-mode speed upstream of the shock, and green dash-dotted lines indicate the $95 \%$ confidence level. 
also present (Kahler 2001). The present analysis shows only a median correlation $(r \sim 0.6)$ between the shock speed (in the solar wind frame) and the SEP fluxes of higher energy

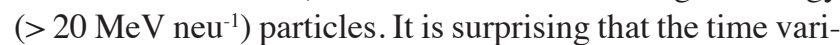
ations of the SEP fluxes followed closely with the temporal variations of the modeled Mach number. For example, in the particular event (FFS3) that started on day 301, a sudden decrease in the shock Mach number caused by collision of a RFS (RFS1 + 2) with a FFS (FFS3) on day 302. The sharp drop in Mach number coincides with the sudden drop (Fig. 1a) in SEP particles of higher energies, indicating the importance of the temporal and spatial evolution of CMEdriven shocks in the time-intensity of SEP profile at $1 \mathrm{AU}$.

The two studied SEP events with their associated CMEs originating near central meridian (Mewaldt et al. 2005) probably contributed to the good correlation result obtained from this study. Although, the correlation length of energetic particles $(\sim 0.1-0.2 \mathrm{MeV})$ at $1 \mathrm{AU}$ is found to be $\sim 3 \times 10^{8} \mathrm{~km}$ (Neugebauer and Giacalone 2005), the prompt rise (within $1 \mathrm{hr}$ ) of high-energy SEPs at the flare onset suggests that the CME-driven shocks, which were developed at the surface of the Sun at the flare onset, accelerate particles to $10 \mathrm{~s}$ of $\mathrm{MeV}$ within $\sim 10 R_{s}$ and are magnetically connected to ACE, and cross-field transport of the accelerated particles are probably not important for the two events. This may also have helped the comparison of the modeled shock using the $1.5 \mathrm{D}$ simulation code. On the other hand, as suggested by Reames (2009), the fast $\left(>2500 \mathrm{~km} \mathrm{~s}^{-1}\right)$ halo-CME that occurred on 28 October 2003, can travel $2 R_{s}$ in a short time (10 minutes) and reach field lines that are well-connected to the Earth, thereby energetic particles that are accelerated by the CME-driven shock can take little time to the observer at Earth. One must note this simplified approach using the 1.5D simulation leaves out a few parameters that are known to affect SEP fluxes. For example, the angle between IMF and the shock normal $\left(\theta_{B N}\right)$. The $\theta_{B N}$ has been associated with the efficiency of diffusive shock acceleration; whether particle acceleration is more efficient in a quasi-perpendicular shock (e.g., Jokipii 1987) or in a quasi-parallel shock (e.g., van Nes et al. 1984) is still a matter of debate. It is not clear whether a better correlation would result if the events are parametrized by the $\theta_{B N}$ angle because it will require at least a $2 \mathrm{D}$ simulation. Nonetheless the present study should represent the lower bound for this type of study. Another relevant parameter is adiabatic deceleration, that could be taken into account in a more complete simulation.

Finally, it is worth mentioning that the correlation analysis between SEP fluxes and shock Mach numbers reveals an energy dependent feature. The cross correlation between the two parameters sharply increases and becomes significant at the $5 \%$ probability level occurs for both species with $E>\sim 10 \mathrm{MeV} \mathrm{n}^{-1}$ (up to the SIS energy), though we are not certain if better correlations can occur for higher energy SEPs. The energy corresponding to the correlation peak is in good agreement with the SEP spectra break reported by Mewaldt et al. (2005) $\left(\sim 22 \mathrm{MeV} \mathrm{n}^{-1}\right.$ for ${ }^{4} \mathrm{He}$ and $\sim 15 \mathrm{MeV} \mathrm{n}^{-1}$ for $\mathrm{O}$ ). It is possible that lower energy particles are prone to scattering by magnetic turbulence upstream to the shock created by shock-accelerated particles (e.g., Lee 1997 , and references therein). Only those particles with high enough rigidity can leak out and be observed far away from the shock (Cohen et al. 2005). Further investigation with more event studies would help justify this point of view.

\section{CONCLUSIONS}

To summarize, we have shown, for the first time, a good linear relationship between the time-intensity profile of ${ }^{4} \mathrm{He}\left(E>\sim 10 \mathrm{MeV} \mathrm{n}^{-1}\right)$ and $\mathrm{O}\left(E>\sim 10 \mathrm{MeV} \mathrm{n}^{-1}\right)$ and the time profile of concurrent fast shock Mach number derived from an 1.5D MHD simulation for two SEP events that occurred on 28 - 31 October 2003. A simplified scatterfree propagation method along the Sun-Earth line is used to correct the delay time, which improves the correlation only slightly. The overall correlation may further improve if additional parameters are considered, but it is at least possible that the contribution of the Mach number will decrease some if another parameter (e.g., shock angle) is also found to be important. Nevertheless, this study provides a strong argument for acceleration by the CME-driven shocks (cf. Dryer 1994) and suggests a possibly good start on further studies toward a forthcoming better understanding of the way high Mach number shocks are able to produce strong gradual SEP events. The present work also clearly demonstrates the importance of the temporal and spatial evolution of CMEdriven shocks in the time-intensity of a SEP profile.

Acknowledgements We would like to acknowledge E. Stone, who is the Principal Investigator of the ACE/SIS instrument team. We acknowledge the use of flare data from the NOAA Space Weather Prediction Center. The work of K. Liou was supported by NSF AGS-0964396 Grant. The work of C. C. Wu was supported by ONR. The work of S. Plunkett was supported by NRL 6.1 Program. The work of S. T. Wu is supported by AFOSR Grant FA9550-07-1-0468 and NSF Grant ATM0754278 to UAH. The work of G. M. Mason was supported by NASA grants NNX10AT75G and NNX07AP69G. The work at Caltech was sponsored by NASA grant NNX8AI11G.

\section{REFERENCES}

Berdichevsky, D. B., D. V. Reames, C. C. Wu, R. Schwenn, R. P. Lepping, R. J. MacDowall, C. J. Farrugia, J. L. Bougeret, C. Ng, A. J. Lazarus, 2009: Exploring the global shock scenario at multiple points between sun and earth: The solar transients launched on January 1 
and September 23, 1978. Adv. Space Res., 43, 113-119, doi: 10.1016/j.asr.2008.03.026. [Link]

Cohen, C. M. S., E. C. Stone, R. A. Mewaldt, R. A. Leske, A. C. Cummings, G. M. Mason, M. I. Desai, T. T. von Rosenvinge, and M. E. Wiedenbeck, 2005: Heavy ion abundances and spectra from the large solar energetic particle events of October-November 2003. J. Geophys. Res., 110, A09S16, doi: 10.1029/2005JA011004. [Link]

Desai, M. I., G. M. Mason, M. E., Wiedenbeck, C. M. S. Cohen, J. E. Mazur, J. R., Dwyer, R. E. Gold, S. M. Krimigis, Q. Hu, C. W. Smith, and R. M. Skoug, 2004: Spectral properties of heavy ions associated with the passage of interplanetary shocks at 1 AU. Astrophys. J., 611, 1156-1174, doi: 10.1086/422211. [Link]

Dryer, M., 1994: Interplanetary studies: Propagation of disturbances between the Sun and the magnetosphere. Space Sci. Rev., 67, 363-419, doi: 10.1007/BF007560 75. [Link]

Dryer, M., Z. Smith, C. D. Fry, W. Sun, C. S. Deehr, and S.I. Akasofu, 2004: Real-time shock arrival predictions during the "Halloween 2003 epoch". Space Weather, 2, S09001, doi: 10.1029/2004SW000087. [Link]

Ebisuzaki, W., 1997: A method to estimate the statistical significance of a correlation when the data are serially correlated. J. Climate, 10, 2147-2153, doi: 10.1175/15 20-0442(1997)010<2147:AMTETS>2 .0.CO;2. [Link]

Ellison, D. C. and R. Ramaty, 1985: Shock acceleration of electrons and ions in solar flares. Astrophys. J., 298, 400-408, doi: 10.1086/163623. [Link]

Gopalswamy, N., S. Yashiro, A. Lara, M. L. Kaiser, B. J. Thompson, P. T. Gallagher, and R. A. Howard, 2003: Large solar energetic particle events of cycle 23: A global view. Geophys. Res. Lett., 30, 8015, doi: 10.10 29/2002GL016435. [Link]

Jokipii, J. R., 1987: Rate of energy gain and maximum energy in diffusive shock acceleration. Astrophys. J., 313, 842-846, doi: 10.1086/165022. [Link]

Kahler, S. W., 2001: The correlation between solar energetic particle peak intensities and speeds of coronal mass ejections: Effects of ambient particle intensities and energy spectra. J. Geophys. Res., 106, 20947-20955, doi: 10.1029/2000JA002231. [Link]

Kahler, S. W., E. Hildner, and M. A. I. Hollebeke, 1978: Prompt solar proton events and coronal mass ejections. Sol. Phys., 57, 429-443, doi: 10.1007/BF00160116. [Link]

Lee, M. A., 1997: Particle acceleration and transport at CME-driven shocks. In: Crooker, N., J. A. Jocelyn, and J. Feynman (Eds.), Coronal Mass Ejections, Geophys. Monog. Ser. 99, 227-234, AGU Press, Washington, DC, doi: 10.1029/GM099p0227. [Link]

Mason, G. M., R. E. Gold, S. M. Krimigis, J. E. Mazur, G. B. Andrews, K. A. Daley, J. R. Dwyer, K. F. Heu- erman, T. L. James, M. J. Kennedy, T. Lefevere, H. Malcolm, B. Tossman, and P. H. Walpole, 1998: The ultra-low-energy isotope spectrometer (ULEIS) for the ACE spacecraft. Space Sci. Rev., 86, 409-448, doi: 10. 1023/A:1005079930780. [Link]

Mewaldt, R. A., C. M. S. Cohen, A. W. Labrador, R. A. Leske, G. M. Mason, M. I. Desai, M. D. Looper, J. E. Mazur, R. S. Selesnick, and D. K. Haggerty, 2005: Proton, helium, and electron spectra during the large solar particle events of October-November 2003. J. Geophys. Res., 110, A09S18, doi: 10.1029/2005JA011038. [Link]

Neugebauer, M. and J. Giacalone, 2005: Multispacecraft observations of interplanetary shocks: Nonplanarity and energetic particles. J. Geophys. Res., 110, A12106, doi: 10.1029/2005JA011380. [Link]

Pallavicini, R., S. Serio, and G. S. Vaiana, 1977: A survey of soft X-ray limb flare images - The relation between their structure in the corona and other physical parameters. Astrophys. J., 216, 108-122, doi: 10.1086/155452. [Link]

Panitchob, S., 1987: An adaptive grid finite difference method for time-dependent magnetohydrodynamic (MHD) flows and its astrogeophysical applications. Ph.D. Dissertation, Department of Mechanical Engineering, University of Alabama, Huntsville, Alabama, USA.

Reames, D. V., 1999: Particle acceleration at the Sun and in the heliosphere. Space Sci. Rev., 90, 413-491, doi: 10.1023/A:1005105831781. [Link]

Reames, D. V., 2009: Solar release times of energetic particles in ground-level events. Astrophys. J., 693, 812821, doi: 10.1088/0004-637X/693/1/812. [Link]

Stone, E. C., C. M. S. Cohen, W. R. Cook, A. C. Cummings, B. Gauld, B. Kecman, R. A. Leske, R. A. Mewaldt, M. R. Thayer, B. L. Dougherty, R. L. Grumm, B. D. Milliken, R. G. Radocinski, M. E. Wiedenbeck, E. R. Christian, S. Shuman, and T. T. von Rosenvinge, 1998: The solar isotope spectrometer for the advanced composition explorer. Space Sci. Rev., 86, 357-408, doi: 10.1023/A:1005027929871. [Link]

van Nes, P., R. Reinhard, T. R. Sanderson, K.-P. Wenzel, R. D. Zwickl, 1984: The energy spectrum of 35- to 1600$\mathrm{keV}$ protons associated with interplanetary shocks. $J$. Geophys. Res., 89, 2122-2132, doi: 10.1029/JA089iA 04p02122. [Link]

Wild, J. P., S. F. Smerd, and A. A. Weiss, 1963: Solar bursts. Annu. Rev. Astron. Astrophys., 1, 291-366, doi: 10.1146/annurev.aa.01.090163.001451. [Link]

Wu, C. C., J. K. Chao, S. T. Wu, and M. Dryer, 1996: Numerical simulation of slow shocks in the solar wind. Sol. Phys., 165, 377-393, doi: 10.1007/BF00149720. [Link]

Wu, C. C., S. T. Wu, M. Dryer, C. D. Fry, D. Berdichevsky, Z. Smith, T. Detman, N. Gopalswamy, R. Skoug, T. Zurbuchen, and C. Smith, 2005: Flare-generated shock 
evolution and geomagnetic storms during the "Halloween 2003 epoch": 29 October to 2 November. J. Geophys. Res., 110, A09S17, doi: 10.1029/2005JA011011. [Link]

Wu, C. C., X. S. Feng, S. T. Wu, M. Dryer, and C. D. Fry, 2006: Effects of the interaction and evolution of interplanetary shocks on "background" solar wind speeds.
J.Geophys.Res., 111, A12104, doi: 10.1029/2006JA01 1615. [Link]

Wu, C. C., S. T. Wu, M. Dryer, C. D. Fry, D. Berdichevsky, Z. Smith, and T. Detman, 2007: The evolution of shocks near the surface of Sun during the epoch of Halloween 2003. J. Atmos. Sol.-Terr. Phys., 69, 91-100, doi: 10.1016/j.jastp.2006.07.014. [Link] 